\title{
Strategies to link rural areas to European and national transport networks
}

\author{
Dana Sitányiová ${ }^{1, *}$, Soňa Masarovičová ${ }^{2}$, Daniele Berselli ${ }^{3}$, Antonio Nicolini $^{4}$ \\ ${ }^{1,2}$ University of Žilina, Faculty of Civil Engineering, Univerzitná 8215/1, 01026 Žilina, Slovakia \\ ${ }^{3,4}$ Agency for mobility and local public transport Modena S.p.A., 41122 Modena, Strada Sant'Anna \\ 210, Italy
}

\begin{abstract}
Transport capacity is very important indicator of country's economic power reflecting its economic development. All means of transport must be efficient and able to transport goods and people as cheaply and quickly as possible. This requires constant upgrading of transport networks often with limited budget. This is a problem especially in rural areas, where population density is very low and there are difficulties in providing transport links between cities and rural communities. It is very expensive to upgrade transport network and also to ensure public transport services there. Individual car transport can diminish the problem, but some groups (the young, old or poor) will always require public transport service, moreover increasing car traffic put pressure especially on existing road transport. Public transport linking isolated rural regions to transport hubs towards the European and national transport networks play a crucial role in that regard. Paper presents some outputs of RUMOBIL project focusing on pilot actions testing a number of innovative applications during a period 2017-2018 how sparsely populated peripheral areas can be better linked to a primary, secondary or tertiary transport node by public transport.
\end{abstract}

\section{Introduction}

Paper presents some of the specific mid-term results of RUMOBIL project, funded by INTERREG CENTRAL EUROPE program. The main objective of RUMOBIL is to improve public transport in rural areas by better connecting them to the national and European transport networks. Project supports transnational cooperation between eight public authorities from Germany, Czech Republic, Poland, Hungary, Slovakia, Croatia and Italy, and their transport entities. These are confronted with a similar challenge to respond to pressures on regional public transport systems caused by demographic change in peripheral areas. Quicker and more comfortable access by public transport positively contributes to the quality of life in rural areas and supports business activities there. To that aim, a set of evidence-based policy recommendations - RUMOBIL strategy - will be elaborated at the end of the project in 2019 to improve the capacities of local and regional

\footnotetext{
* Corresponding author: dasit@fstav.uniza.sk
} 
decision-makers responsible for the design and coordination of public passenger transport in Central European regions affected by demographic change, providing insights in already proven good practices and novel solutions. Main outputs of RUMOBIL project are also pilot actions, and policy-decisions to implement the RUMOBIL strategy in the eight partner regions through an improvement of their transport plans. Eight pilot actions have allowed testing a number of innovative applications during a period 2017-2018. One of the pilots is introduced in this paper in more detail as example of best practice.

\section{Necessity to contrast the population decreasing}

European rural areas are facing specific challenges, including large trip distances, scattered populations, a high proportion of elderly people, a high level of poverty and unemployment in some regions and often also remoteness from the main transport infrastructure. Nowadays, rural areas are about the half of entire European territory and they are occupied by the $20 \%$ of the total population, so it is clear how this is a matter of a certain relevance. [1] Such a huge number of people, therefore, lives in a situation that is definitely different from what is considered the standard quality of life for the rest of Europe. This condition is characterised by several aspects that added up lead to a specific scenario. First of all, the main issue affecting rural areas is the demographic change (strictly connected to the distribution of activities).

If we compare population change in predominantly urban and rural region, we can see differences there (figure 1, figure 2) Ageing of population in Europe is going to look very different in urban and in rural areas. While both urban and rural areas in Europe are witnessing a rapid increase of their pension-age populations, the picture looks very different for working-age populations. While many urban areas are seeing also an increase of their working-age populations, rural areas are often faced with a decline of their working-age populations. [2]

Business cores, workplaces, universities, etc. are always more concentrated in big cities and, in this way, rural inhabitants (in particular, working-age and young) have to make a decision: move towards the city or stay in the village trying to reach their own destination anyway. It is not rare to see the first case, with an obvious result: in rural areas the average age clearly grows, entire families leave their original territory so that elderly people become the main part of the population.

This leads to another issue of rural areas: in certain sites it can be observed a lower population density than expected, with the consequence that these areas become even more isolated. All is reflected in another issue, i.e. the poor Public Transport (PT) offer. In areas with low densities of population it is more difficult to realise a proper PT service since the demand is not enough and it would lead to an under-utilised system, with enormous costs. Obviously, this is a further reason behind the movement towards the city. [1]

Many people cannot imagine their life without car because even in the relatively densely populated regions of Europe, rural areas tend to lack public transport and is thus a need for alternative public transport services to support car-free mobility in rural areas. Innovative schemes such as flexible or on demand transport services may be economically viable solutions. They can be efficient while at the same time environmentally friendly.

Infomobility concerns a lot of fields, all important to make a service more attractive and, above all, simple. Many of them refer to the matter of real-time information (which is for sure very heartfelt by users) that can really improve the perceived quality of a service. Other actions focus on helping people in their trip planning, to make useful decisions, while other propose to manage booking services and ticket buying to make users save time and troubles. 


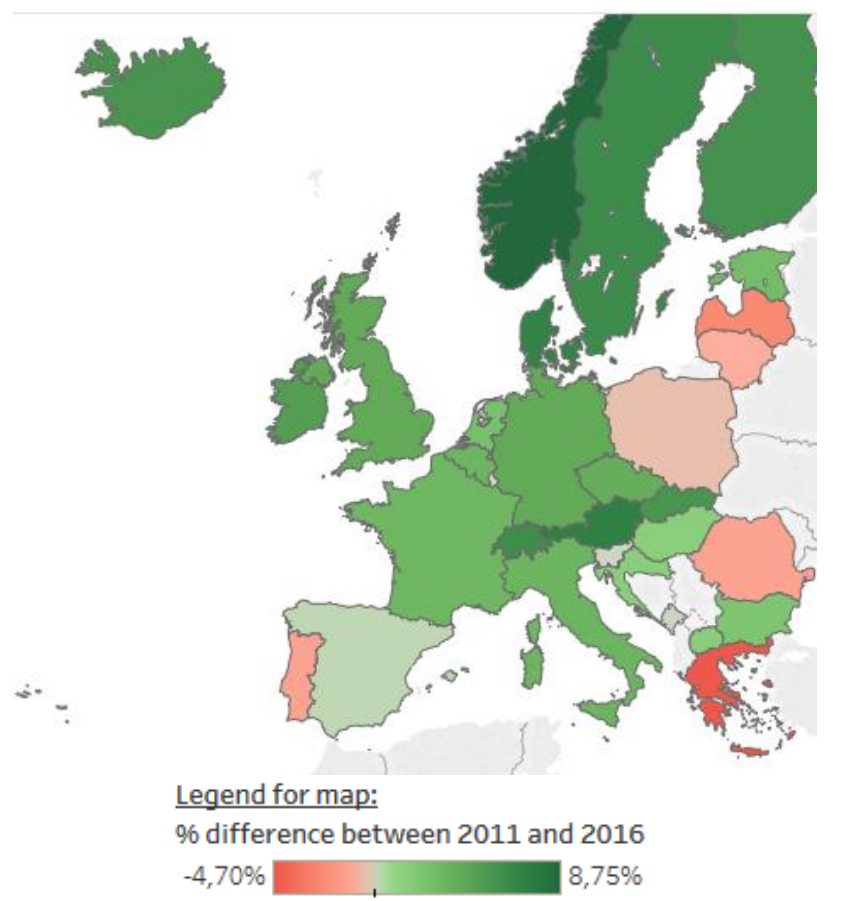

Fig. 1. Population change between 2011 and 2016 (in \%) in predominately urban regions, Source: Eurostat, [1]

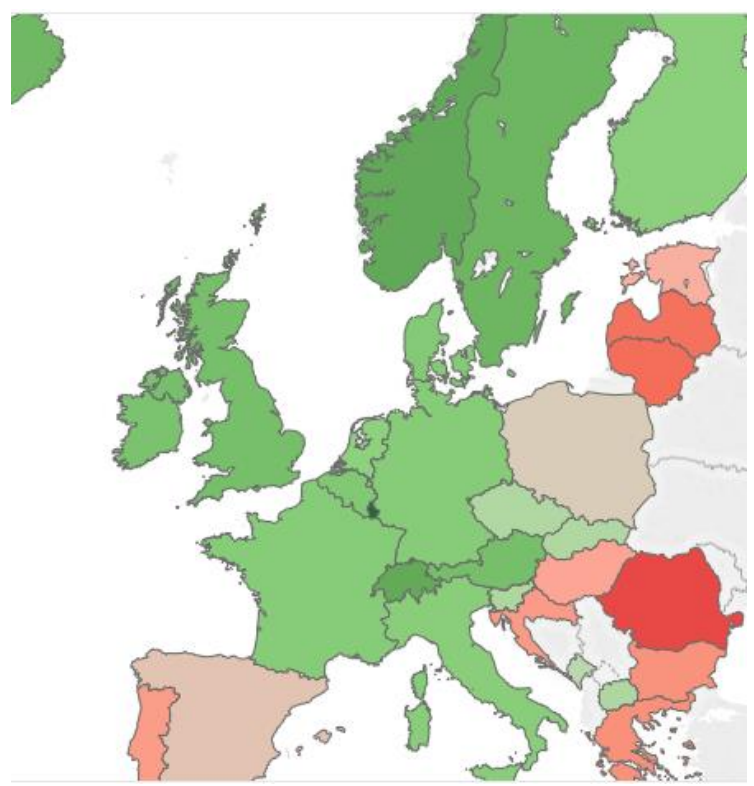

\section{Legend for map:}

\% difference between 2011 and 2016

$-7,72 \%$ $12,58 \%$

Fig. 2. Population change between 2011 and 2016 (in \%) in predominately rural regions, Source: Eurostat, [1] 


\section{Pilot projects}

\subsection{Pilot topics and monitoring indicators}

Pilot activities were implemented in eight partner European rural regions concerned at least one of the following project topics:

- New services to better connect rural areas (e.g. new bus services, solutions to improve the inter-modality, new rail services);

- Enhanced passenger information to increase the quality of public transport;

- Improvement of access points to national and EU transport network (e.g. upgrading of train and bus stations).

Project partners include different types of organisations, however also external stakeholders - parties (organisations, individuals) that have an interest in a project, and can either affect or be affected by the mobility measure were addressed to participate in each pilot. Citizens and stakeholders involvement was a precondition for successful pilots, since long-term perspective of measure requires a high degree of support and acceptance. The main project stakeholder is a group of residents who are awaiting the arrival of a better transportation system and services in the region. [3]

Since spring 2017 partners have been performing pilot projects in selected areas as experimental trials, small-scale, short-term projects, which can help them to learn how a larger-scale project might work in practice, in future, in whole region. In this way organizations can test measure, prove its value, feasibility and reveal deficiencies before spending a significant amount of time, energy or money on a larger-scale project.

Due to fact that there are various types of pilots, the common comparable set of 50 obligatory indicators was defined. Geographical indicators describe the location where the pilot action takes place, which can be useful to understand spatial implications, relationship between urban and rural environment etc. Demographic and social factors are very influential on the public transport demand. Without understanding the transport system dynamics and more importantly, the behaviour of public transport travellers, it would be difficult to make accurate transport demand forecasts, which are necessary for service planning, marketing, and fare policy purposes. Operational or performance indicators can support public transport planning in rural areas in many ways and allow to determine if resources were used efficiently and equitably, identify potential problems and wins, and to verify whether a particular pilot achieves its predicted targets. Selected economic indicators reflect to the costs of pilot providing information about the feasibility of the pilot and also about its effectiveness during operation. Qualitative indicators describe the level of satisfaction with a new or existing public transport service and allow passengers to evaluate pilot projects.

\subsection{Pilot project in Modena region}

The pilot project developed by Agency for mobility and local public transport Modena S.p.A. - aMo, has been performed in the town of Castelfranco Emilia in Province Modena, Italy, where a demand-responsive transport (DRT) service called Prontobus has been active since 2006. The town is located east of Modena (about $13 \mathrm{~km}$ along); it has a population of 32,174 inhabitants (2014), an area of $102.47 \mathrm{~km}^{2}$ with a density of 304 inhabitants $/ \mathrm{km}^{2}$. Castelfranco Emilia hosts basic services, a health district as well as a railway station of the main regional and national network that allows easy connection to the provincial and regional capitals and all major Italian cities. 
The territory of the municipality is significantly extended and includes 8 smaller villages that insist on rural areas as the local economy is still based largely on agriculture. The proximity to the cities of the provincial (about $13 \mathrm{~km}$ ) and regional capital (about $25 \mathrm{~km}$ ), with a lower cost of houses over the major cities and the presence of public services, has fostered a strong demographic growth in the last 25 years, even if significant economic and productive activities are not presented in the area.

The population in fact has grown from about 21.000 inhabitants in 1991 to over 32.000 today, with an increase of $52 \%$; the most of these (about $70 \%$ ) went to live in the small villages and rural areas around Castelfranco Emilia. This has generated a repopulation of rural areas that were gradually abandoned during the sixties when it was prevalent the attraction of the city or the capital cities. This reversal of the demographic trend towards the smaller villages and rural areas highlighted the need to provide transport services that would enable the connection to the main city and most important primary services.

The capillarity of traditional public transport services is not enough to represent a suitable response to the needs of mobility of the rural area's inhabitants. This aspect has generated the need to activate a DRT service that is more appropriate for this kind of areas. DRT service typically provides a PT service for areas of low passenger demand, where a regular bus service would not be viable. The DRT service Prontobus (figure 3) was created with the objective of ensuring this need for primary mobility in a large dispersed area substantially without ordinary transport services. In a period of economic resources continuously declining it is essential to increase the number of passengers to make the PRONTOBUS service economically sustainable. [4]

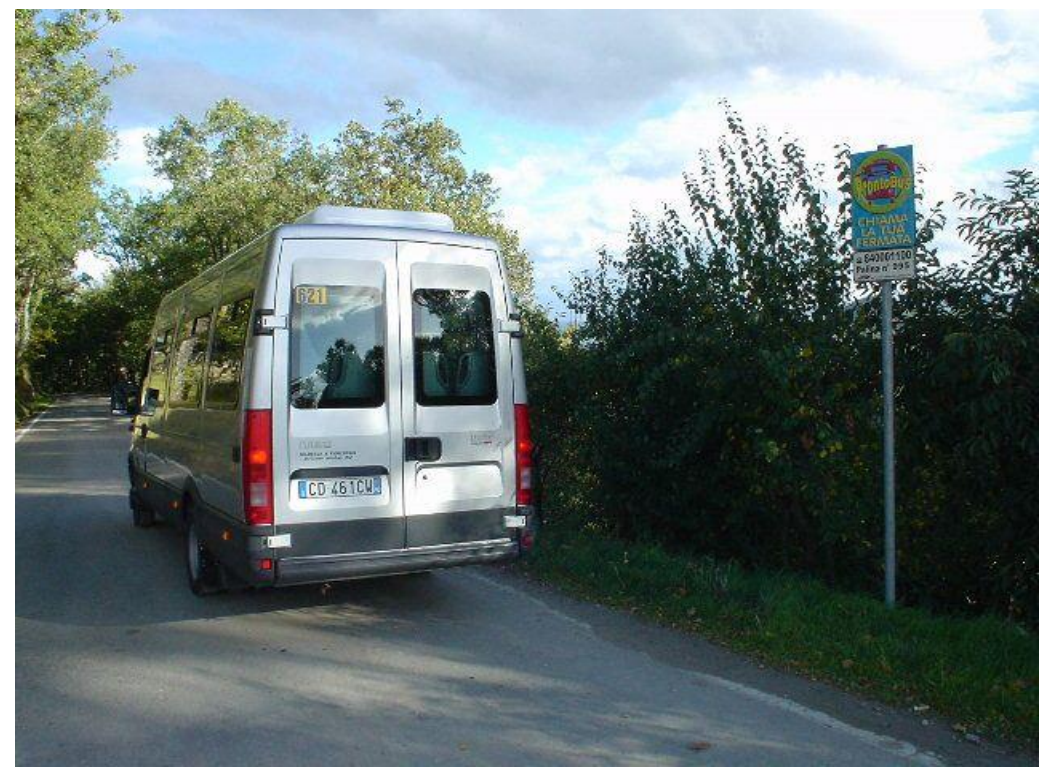

Fig. 3. Prontobus leaving bus stop, Source: [4]

Prontobus is performed from the public transport Operator for the Province of Modena and is available from Monday through Friday, excluding holidays, from 9.00 to 12.00 and from 14.30 to 17.30 (19.30 for travels within the area of Castelfranco Emilia). The service does not take place in August and it is possible to enter the service with the same tickets and season cards that are used for ordinary public transport services with free interchange between the two kind of services (ordinary and on demand). 
Before implementation of RUMOBIL pilot the service was available with reservations that were made through a call center. The call center handled reservations manually and no information about service was provided to users. Prontobus carried about 10.000 passengers a year - about 3 travellers per trip, mainly used for connections with the train station and the hospital. The service was underused mainly due to the lack of knowledge and the difficulty of access to information on the service. The lack of information about the provision of the DRT service has been highlighted by users - through a phone survey - as one of the main difficulties in accessing it.

The pilot action has been implemented to provide better knowledge of the service to increase the number of users and thus give greater visibility to a fundamental service to prevent the depopulation of rural areas around the city of Castelfranco Emilia. Due to the nature of DRT services, no timetables were available for users and the only way to know something about the service's scheduling was to call the call center. A new dedicated software was developed. The system consists of:

- Web portal (www.rumobil-modena.eu) for the users with information about the Prontobus service and the situation of reservations updated in real time with the possibility to register in order to receive mails or SMS about interesting services recognized for the day after;

- Web site dedicated to the management of reservations for the call center;

- App for users of the Prontobus service to view reservations updated in real time and with the ability to book an existing trips directly from the app;

- App for drivers of the Prontobus service to get information about reservation in real time and the trips to be made.

aMo has developed procedures to upload all data recorded by the call center to a dedicated database. Using a reporting tool, it is possible to have detailed information that was previously unknown (figure 5).

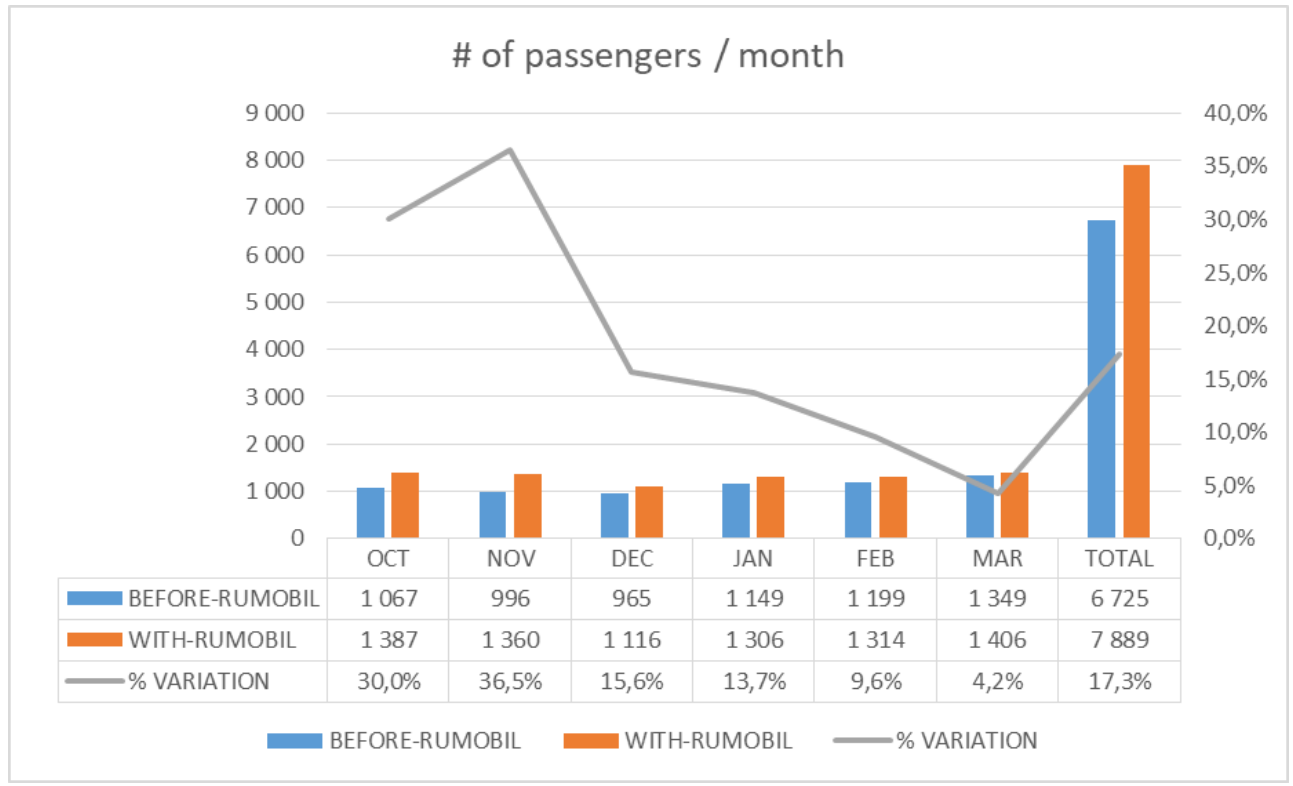

Fig. 4. Number of passengers before and after RUMOBIL pilot implementation, Source: [5]

Before the introduction of the RUMOBIL measure, the only data available was the number of travellers per day, while now it is possible to know how these trips have been made in detail (travel time, origin-destination of the trip, etc.). The increase in travellers 
(figure 4) registered after the activation of the RUMOBIL measure was significant and exceeded expectations providing valuable information to be used for the definition of the RUMOBIL strategies. [5] Positive feedback came from the primary stakeholders, the users of the Prontobus service, who reported appreciation in particular for having real-time reservations available. Another good result is for the call center that has improved the management of reservations and is very satisfied with the introduction of the RUMOBIL system.

They are also requiring the extension of the RUMOBIL system to the other regions operated by Prontobus service to improve their operation. The drivers expressed satisfaction with the introduction of the RUMOBIL system as they now receive the information faster and more safely and are no longer distracted from driving by the calls received from the call center, if there are any changes in the bookings. Through a reporting tool it is now possible to elaborate detailed statistics (figure 5) that was not available before; this information is indispensable in order to be able to intervene in the services for a better tuning.

\# TRIPS / ORIGIN-DESTINATION
\begin{tabular}{|c|c|c|}
\hline ORIGIN & DESTINATION & \# TRIPS \\
\hline CE01 & CE33 & 1 \\
\hline CE01 & CE34 & 1 \\
\hline CE03 & CE31 & 3 \\
\hline CE03 & CE32 & 34 \\
\hline CE03 & CE33 & 141 \\
\hline CE03 & CE34 & 6 \\
\hline CE04 & CE32 & 19 \\
\hline CE04 & CE33 & 5 \\
\hline CE04 & CE34 & 1 \\
\hline
\end{tabular}

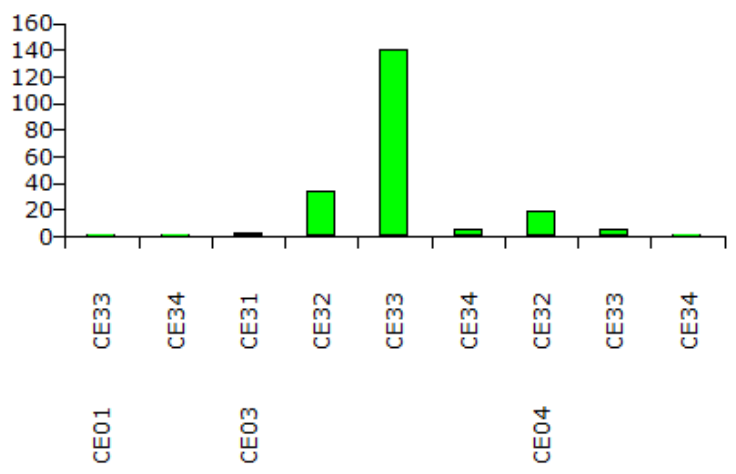

Fig. 5. Example of reporting, number of trips per origin-destination stops, Source: [5]

It has to be underlined that the system is constantly updated and evolved; if new needs and additional functionalities are requested by the users, by the call center or by the drivers and if these are evaluated positively, those are introduced into the system by the system developer. The data collection represents a new and more reliable method to draw demand patterns, since they could be based on real decisions. Similarly, by enhancing infomobility, people could feel more at ease using PT, because there will be a constant and real time support that could avoid unpleasant situations (delay, mistakes, etc.) making the trip more 
enjoyable and attractive. In addition to these, services like this DRT can be really a notable innovation, since they meet the needs of those who otherwise would have no other choice.

\section{Conclusions}

The final RUMOBIL project deliverable is RUMOBIL Strategy, a work paper that highlights the necessity to link rural areas to European and national transport networks and shows how this can be accomplished with the limited resources available. Furthermore, the RUMOBIL Strategy is the step thanks to which the solutions that could be accepted by the decision makers for the improvement of the sustainable mobility in their own rural and peripheral areas have pointed out.

As expected, the most common objective among the pilots is to increase the use of PT in rural areas, also considering the introduction of new services or more flexible ones. The enhancement of the existing PT systems is based on the improvement of basic features (timetable, integrated tariffs, friendliness of vehicles and stations/stops, new and better interchange terminals) and in particular on the promotion of infomobility, that plays a key role in all the layers (on board, at the stops, on users' devices). What emerges from the all pilots is that no result can be achieved without a proper and well done communication campaign that allow all the potential users to be aware of their opportunities beyond private cars.

In terms of transnationality and transferability, the Strategy will allow other regions' transport actors to clarify their expectations concerning the objectives and solutions of RUMOBIL project and, in more general terms, of the improvement of the mobility in rural areas. Finally, the Strategy is based on a common starting point that is composed mainly of comparative and unique analysis of good practices and lessons learnt, pilot test results and recommendations.

This paper is published within the implementation of the project RUMOBIL (CE55), co-funded by European Regional Development Fund under Interreg CENTRAL EUROPE.

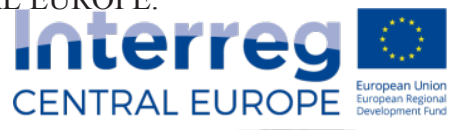

\section{RUMOBIL}

\section{References}

1. RUMOBIL consortium, Report on the strategies to link rural areas to European and national transport networks (2018)

2. Koessl, G. Demographic change in Europe: urban vs rural. (2018, May 16). Retrieved from https://public.tableau.com/profile/gerald.koessl\#!/vizhome/demographicchange/in Europe

3. RUMOBIL consortium, Stakeholder involvement report (2017)

4. Berselli, D. D.T2.4.4 Pilot launch report - Castelfranco Emilia (2017)

5. Berselli, D. D.T2.4.5 Pilot interim report-Castelfranco Emilia (2018) 